\title{
Comparison of a low-frequency Butterworth filter with a symmetric SE-filter
}

\author{
K S Medvedeva ${ }^{1}$ \\ ${ }^{1}$ Saratov State University, Astrakhanskaya Street 83, Saratov, Russia, 410012
}

\begin{abstract}
The article compares two filters: a Butterworth filter and an asymmetric SEfilter.The experimental studydetermines their advantages and disadvantages. Also,experiments based on the peak signal-to-noise ratio(PSNR) metricshowvisual evaluation. The results of experimentsshow that a symmetric filter better restores images usinga small set of continuous function parametersthatare distorted by a low-frequency Gaussian filter.
\end{abstract}

\section{Introduction}

Due to the imperfection of forming and recording systems, images recorded by systemsare distorted (fuzzy) copiesof the original images. The main causes of distortions that resultindegradation of clarity includethe limited resolution of the forming system, refocusing, the presence of a distorting medium (for example, the atmosphere), and movement of the camera on the object being registered. Eliminating or reducing distortion for clarity is the task of image recovery.

Automatic control systems, measuring equipment, signal processing systems, and various filters with different characteristics are used to filter signalsin telecommunications. Depending on the frequency band associated with the bandwidth and the suppression band, there are odd, band, highfrequency, and low-frequency filters. Also, all-pass filters have a constant amplitude-frequency response in the required frequency range, and their phase-frequency response is a given frequency function [1].

The simplest way to restoreimage clarity is to process the observed image in the spatial frequency domain with an inverse filter [2].The drawbacks of this filter are the occurrence of edge effects, which take the form of an oscillating hindrance of high power that completely masksthe reconstructed image. Boundary effects can be observed even in noise-free images. But the main problem ofusing an inverse filter is that the corresponding transfer function can have poles close to zero.

This paper considers two frequency filters: a Butterworth filter and a symmetric SE-filter [3]. By means of experiments, comparative analysis will be carried out to identify their advantages and disadvantages. In paper [3], the technology of FIR filter synthesis is proposed, in which identification of the parameters of a continuous function approximating the impulse response is carried out instead of determining the impulse response samples. In this case, the parametric family of approximating functions, depending on a small number of parameters, is given, taking into account the desired frequency characteristics of FIR filters intended for distortion correction.

The Butterworth filter is chosen for several reasons. The amplitude response of this filter is smooth at the frequencies of the passband and almost decreased to zero at the frequencies of the suppression band. The Butterworth filter is the only filter that retains the amplitude-frequency response for higher 
orders (except for the steep drop in the characterof the suppression band), whereasmany other types of filters (Chebyshev filter, Bessel filter, elliptical filter) have different forms of frequency response in different orders. In [3], a symmetric filter with a Wiener filter was compared. This paperpresents the results of an experimental comparison ofa symmetric filter and a low-frequency Butterworth filter.

\section{Statement of the problem}

The transfer function of a low-frequency Butterworth filter (SFNF) of order $\mathrm{n}$ with a cutoff frequency at a distance $D_{0}$ from the point of origin is written by the formula [4]:

$$
H(u, v)=\frac{1}{1+\left[D(u, v) / D_{0}\right]^{2 n}} .
$$

where the distance $D(u, v)$ is given by the formula

$$
D(u, v)=\left[(u-M / 2)^{2}+(v-N / 2)^{2}\right]^{1 / 2} .
$$

The transfer function of the CFFF does not have a discontinuity, which establishes an exact boundary between the transmitted and cut frequencies. For filters with a smooth transfer function, it is common practice to determine the location of truncating frequencies as a set of points, where the values of the function $H(u, v)$ become less than some of its largest value. In the case of the Butterworth function, $H(u, v)=0,5$ (less than $50 \%$ of the largest value equal to 1 ) for $D(u, v)=D_{0}$.

The Butterworth filter is characterized by the order of filtering, a parameter that determines the steepness of the filter's transfer function. For small filter order values, the transfer function has a smooth shape, close in form to the transfer characteristic of the Gaussian filter; at high values, the filter is characterized by a steep transfer function and approaches the characteristics of an ideal filter in shape.

The advantages of low-frequency Butterworth filters include a lesser manifestation of unwanted blurring effects and the appearance of false circuits compared with an ideal low-frequency filter. As the order of the low-frequency Butterworth filter increases, the effects of blurring also increases.

An experimental comparison of the Butterworth filter with a symmetric SE-filter is interesting. In this paper, a FIR filter with a zero phase was constructed, that is with a real frequency response $[2,5]$. Hence the reference region will bea $N \times N$ square with side $N$ and center in the point with coordinates $k_{1}=0, k_{2}=0, N$ is odd and $N_{1}=N_{2}=N$.

The filter has a reference region $\mathrm{D}$ :

$$
\left\{D\left(n_{1}, n_{2}\right): 0 \leq n_{1}<N_{1}, 0 \leq n_{2}<N_{2}\right\} .
$$

The count of the image $y\left(n_{1}, n_{2}\right)$, reconstructed with the FIR filter, can be determined from the readings $x\left(n_{1}, n_{2}\right) \in D$ of the distorted image of the relation:

$$
y\left(n_{1}, n_{2}\right)=\sum_{k_{1}=0}^{N_{1}-1} \sum_{k_{2}=0}^{N_{2}-1} h\left(k_{1}, k_{2}\right) x\left(n_{1}-k_{1}, n_{2}-k_{2}\right),
$$

where $h\left(k_{1}, k_{2}\right)$ is a two-dimensional impulse response.

To describe the one-dimensional frequency response in the radial direction, Iuse the QEFR filter model [9]:

$$
\begin{array}{r}
S(\omega)=\left\{\begin{array}{l}
a \omega^{2}, \text { npu }|\omega| \leq \omega^{*}, \\
e^{-c|\omega|}, \text { nрu }|\omega|>\omega^{*},
\end{array}\right. \\
S\left(\omega^{*}\right)=\alpha \omega^{* 2}=e^{-c \omega^{*}}
\end{array}
$$

The approximation function of a symmetric SE filter is: 


$$
h(r)=\frac{e^{-c \omega^{*}}}{\pi}\left[\frac{\sin \omega^{*} r}{r}+\frac{2 \cos \omega^{*} r}{r^{2} \omega}-\frac{2 \sin \omega^{*} r}{r^{3} \omega^{* 2}}+\frac{\left(c \cos \left(\omega^{*} r\right)-r \sin \left(\omega^{*} r\right)\right)}{c^{2}+r^{2}}\right]
$$

As (7) shows, the approximation of the impulse response $h(r)$ is reduced by tuning two parameters: $\omega^{*}$ and $c$. Parameter $\omega^{*}$ is the abscissa of the greatest value of the frequency response of the FIR filter. Parameter $c$ characterizes the degree of presence of high frequencies in the reconstructed image.

The advantages of this technology for constructing FIR filters include the possibility of achieving a high quality of image reconstruction by tuning a small number of parameters of a continuous function approximating the impulse response corresponding to a given frequency response.

\section{Results of experiments}

In the Butterworth filter, also to the distance $D_{0}$ outside of which all high-frequency components of the Fourier image, which is set from 0 to 256 , are removed, the filter order from 0 to 20 is additionally specified.

The filter parameters were adjusted using the "Lena" test image (Figure 1).

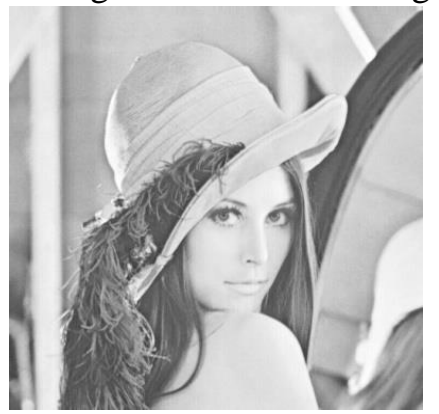

Figure 1. Test drawing "Lena".

Next, the original image was distorted by simulatinga low-pass Gaussian filter for several variants of specifying $\sigma$. The degree of blurring was specified for $\sigma=3$ and $\sigma=5$. Figures 2(a) and 2(b) show a test image of "Lena" after introducing distortions with given parameters.

In this paper, the PSNR (peak-to-noise ratio) metric is used, defined by the formula: $P S N R=10 \log _{10}\left(\frac{M A X_{I}^{2}}{M S E}\right)=20 \log _{10}\left(\frac{M A X_{I}}{\sqrt{M S E}}\right)$, where MSE (mean square error) is the root-meansquare error and $M A X_{I}$ is the greatest value received by the pixel of the image.

The following results were obtained in [3]. Metric PSNR evaluated the results on the test image "Lena". The greatest values of PSNR were 30.41 for $\sigma=3$ and 27.18 for $\sigma=5$. For the next experiment, the test image was distorted with a blur intensity of $\sigma=3$. Additionally, the noise was "superimposed" in the range of 20 to $50 \mathrm{~dB}$. Furthermore, depending on the values of the reference region, comparisons were made ofthe PSNR metric. With the size of the reference region $N=5$, the best result was 29.92. With the increase in the reference region, the metric values decreased. With $N=13$, they were already 26.66 .

Table 1 shows the results of the experiment with a Butterworth filter. The image is distorted by a low-frequency Gaussian filter. Two variants with different degrees of blurring ( $\sigma=3$ and $\sigma=5$ ) are used. In the second column, the size of the reference region $N$ is recorded, in the third, the distance $D_{0}$, behind all components of high-frequency Fourier images, which can be specified in the range from 0 to 256. Column $n$ indicates the order of the filter, which is in the range from 0 to $20 . N$ is the size of the reference area. The last column shows the results of the PSNR metric.

Table 1 shows the results of an experiment with a Butterworth filter and a blur level of $\sigma=3$ and $\sigma=5$. For $\sigma=3$, the greatest result is achieved with a filter with a cutoff frequency of 170 and a 
filter order of 10. For the same cutoff frequency, but with different filter orders, the PSNR metric is changed. With the blur level $\sigma=5$, the best result is achieved with a cutoff frequency of 170 and a filter order of 5 .

Table 1. PSNP with noise in the image "Lena" at $\sigma=3$ and 5 filtered by a Butterworth filter.

\begin{tabular}{cccccccccr}
\hline & $N$ & $D_{0}$ & $\mathrm{n}$ & $P S N R$ & & $N$ & $D_{0}$ & $\mathrm{n}$ & $P S N R$ \\
\hline$\sigma=3$ & 3 & 256 & 20 & 25.02 & $\sigma=\mathbf{5}$ & 3 & 256 & 20 & 26.29 \\
& 5 & 230 & 15 & 26.16 & & 5 & 230 & 15 & 24.88 \\
& 3 & 200 & 10 & 26.14 & & 3 & 200 & 10 & 24.94 \\
& 5 & 170 & 5 & 26.22 & & 5 & 170 & 5 & $\mathbf{2 6 . 4 5}$ \\
& 3 & 170 & 10 & $\mathbf{2 8 . 3 8}$ & & 3 & 170 & 10 & 26.44 \\
& 5 & 170 & 15 & 28.25 & & 5 & 170 & 15 & 26.43 \\
& 3 & 150 & 1 & 26.21 & & 3 & 150 & 1 & 26.18 \\
& 5 & 100 & 20 & 26.19 & & 5 & 100 & 20 & 25.71 \\
& 3 & 50 & 10 & 25.99 & & 3 & 50 & 10 & 26.01 \\
& 5 & 20 & 5 & 25.55 & & 5 & 20 & 5 & 24.64
\end{tabular}

a)



b)

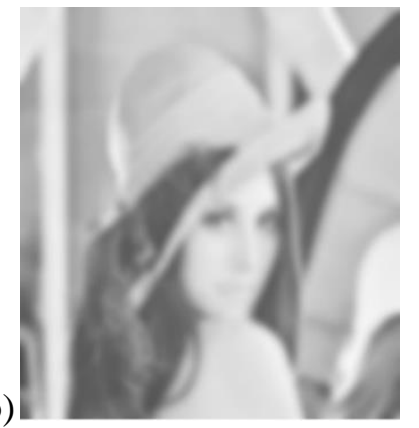

Figure 2. Lena image: distorted with blur (a) $\sigma=3$, (b) $\sigma=5$.

Figure 3 shows the filtered images with the largest PSNR. Figure 3(a) shows the results of a symmetric filter with a blur level of $\sigma=3$. Figure 3(b) shows the results of blurring with $\sigma=5$. Figure 4 shows the results of the experiment with a Butterworth filter.

a)

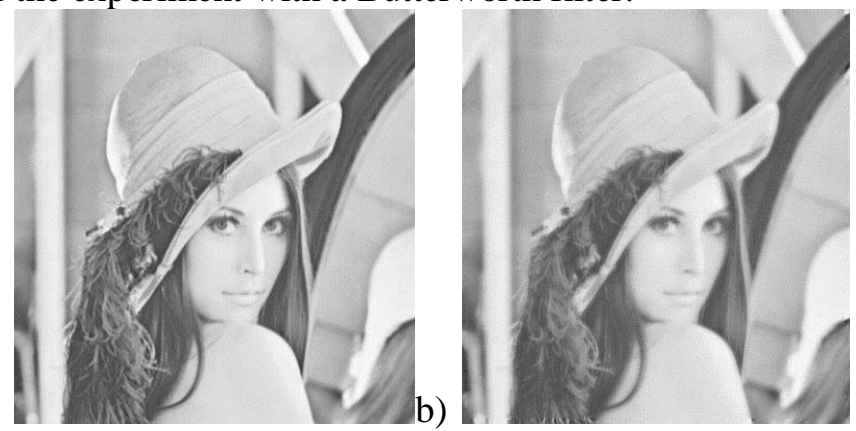

Figure 3. Filtered image of "Lena": radially symmetric SE-filter with blurs(a) $\sigma=3$, (b) $\sigma=5$.

The problem of using the Butterworth filter is that onemust choose the cutoff frequency and the filter order by oneself. Choosing non-optimal values can lead to significant distortions in the filtered image. For example, if onetakes too small a cutoff frequency, most of the minor details of interest will be eliminated. If high values are selected, the processed image will be different from the test image, and the noise level will become insignificant. As the order of the low-frequency Butterworth filter increases, undesirable effects of blurring increase. Also, at very low cutoff frequencies, the so-called "ringing" effect or the Gibbs effect can appear on the filtered images. It is expressed in the appearance of false contours around real ones. 

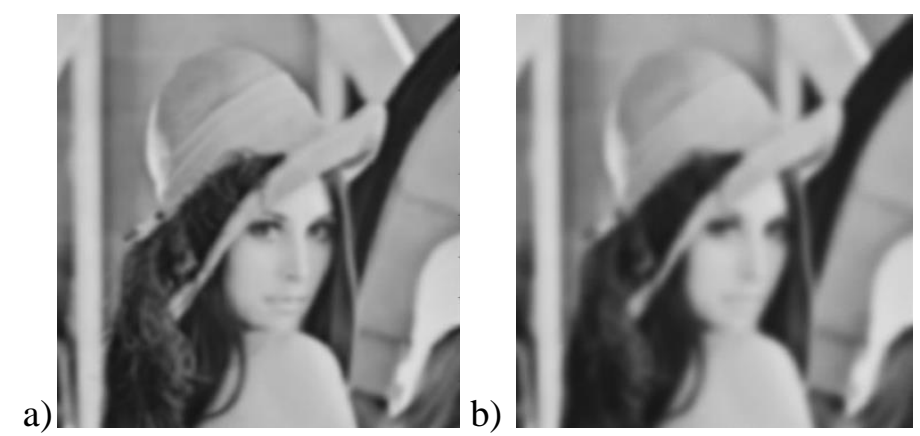

Figure 4. Filtered image of "Lena" by the Butterworth filter with blur (a) $\sigma=3$, (b) $\sigma=5$.

\section{Conclusion}

As a result of these experiments, the following conclusions can be drawn. A radial-symmetric filter has a high quality of distorted imagesrecovery due to the change of a small number of continuous function parameters. Selecting the optimal filtering parameters is difficult when the Butterworth filter is used. It is necessary to conduct a large number of experiments to find the optimal values of the cutoff frequency and the order of the filtration.

Also, a symmetric filter has advantages in quality evaluationby the PSNR metric. The greatest values of this filter were 30.41 for $\sigma=3$ and 27.18 for $\sigma=5$. The Butterworth filter shows the following results: with a distortion ratio $\sigma=3$, the PSNR metric was equal to 28.38; with $\sigma=5$, the greatest result was equal to 26.45 .

A visual assessment of the quality of images processed with a radial-symmetric filter and a Butterworth filter determined thatthe first one coped better with removing the Gaussian blur. Thus, it was found that the use of a radially symmetric SE-filter can provide a quality (by PSNR) superior to the result obtained by using of the Butterworth filter. It is also worth noting that edge effects and blurring, in contrast to the Butterworth filter, are very small in this case.

\section{References}

[1] Sorokin G A 2015 Low-pass filters Computer technologies, management, radio electronics 15 100-107

[2] Pratt W 1982 Digital Image Processing (Moscow: The World) p 480

[3] Fursov V A 2016 Construction of FIR filters in a given parametric class of frequency characteristics for correction of defocusing Computer Optics 40(6) 878-886 DOI: 10.18287/ 2412-6179-2016-40-6-878-886

[4] Gonzalez R and Woods R 2005 Digital Image Processing (Moscow: Technosphere) p1072

[5] Shapiro L and Stockman J 2006 Computer Vision (Moscow: BINOM) p 752

[6] Ziatdinov S I 2015 Pulse characteristic of Butterworth complex b and pass filter Izvestiya Vysshikh Uchebnykh Zavedeniy 5(8) 653-658

[7] Fursov V A, Gavrilov A V, Goshin Ye V and Pugachev K G 2017 Conforming identification of the fundamental matrix in the image matching problem Computer Optics 41(4) 559-563 DOI: 10.18287/2412-6179-2017-41-4-559-563

[8] Glumov N I and Myasnikov E V 2007 Method of selection of informative signs on digital images Computer Optics 31 73-76

\section{Acknowledgement}

The article was supported by Doctor of Technical Sciences, Professor, Head of the Department of Supercomputers and General Informatics at the Samara University Fursov Vladimir Alekseevich. 